\section{The Efficacy of Social Stories in the Classroom to Reduce Disruptive Behaviours in Children with ADHD}

\author{
Charlotte W Greenway* \\ School of Psychology, University of Wales \\ Trinity Saint David, United Kingdom
}

\section{*Corresponding author: \\ Charlotte W Greenway \\ ”c.greenway@uwtsd.ac.uk}

The purpose of this study was to examine the efficacy of Social Stories on the disruptive behaviours of three primary school boys with ADHD. Children's disruptive behaviours were monitored, and observations carried out by Teaching Assistants, before baseline measurements. Social Stories were created to provide children with alternative social responses, which in turn reduce the target behaviours of calling out of turn, rising out of seat and aggressiveness. Following baseline data, each child read their individual stories, and their behaviour was monitored for ten days. After this intervention period, the story was withdrawn and behaviour monitored for a further five days; this allowed the researcher to obtain maintenance data, post-intervention. Results revealed that disruptive behaviours decreased compared to baseline measurements and continued to do so once the story was withdrawn in two out of the three children. Since additional strategies, such as prompts and reinforcement were controlled in this study, the findings have implications for classroom practice. In particular, Social Stories may provide teachers with an alternative strategy to the usual behaviour modification techniques used to control disruptive behaviour in children with ADHD.

Keywords: ADHD; Social stories; Disruptive behaviour; Classroom intervention
Senior Lecturer, School of Psychology, University of Wales Trinity Saint David, College Road, Carmarthen, SA31 3EP, United Kingdom.

Tel: 01267676923

Citation: Greenway CW (2018) The Efficacy of Social Stories in the Classroom to Reduce Disruptive Behaviours in Children with ADHD. Acta Psychopathol Vol.4 No.3:17

Received: May 08, 2018; Accepted: May 18, 2018; Published: May 31, 2018

\section{Introduction}

Attention-Deficit/hyperactivity disorder (ADHD) is characterised by developmentally inappropriate levels of inattention and/or excessive amounts of activity/impulsivity [1]. ADHD is the most prevalent childhood disorder, affecting approximately 4 percent of school-aged children in the UK [2]. Boys diagnosed with ADHD outnumber girls by approximately three to one [3].

Due to the severity of symptoms displayed by children with ADHD, the impact upon their school work and social functioning can be devastating. Research has found that children with ADHD are more likely to receive special educational requirements, receive poorer grades [4] and are at a higher risk of absenteeism and school dropout [5]. ADHD children often struggle in school because of the type of tasks expected of them [6]. The classroom is full of distractions for the ADHD child and attention and maintaining concentration may be difficult due to a lack of motivation and repeated need for appraisal [7]. Even when children are interested in the activity they are engaged in, sustained interest is often short-lived [8], making daily tasks in the classroom difficult for educators to administer. Although there has been much emphasis on the academic difficulties demonstrated by children with ADHD, when primary school teachers were asked about their concerns regarding children with ADHD, they reported the disruptive nature of the disorder was the most significant challenge they faced. The same teachers also reported it as the most detrimental factor to the classroom as a whole [9].

Three key areas of disruption in the classroom associated with ADHD are aggression, rising out of the seat and calling out of turn. All three have been found to be detrimental to the child's academic, cognitive and social functioning in the classroom $[1,10,11]$. According to Barkley [1], aggressive behaviour is present in approximately $40-70 \%$ of school-age children with $A D H D$. The reason behind such high levels may be due to children with ADHD often finding difficulty expressing their emotions, but when they do, they express their feelings more strongly than children without ADHD [10]. Another characteristic exhibited by 
children with ADHD in the classroom is rising out of the seat. The disruptive nature of walking around disturbs other children and is a cause of concern for teachers [11]. Equally disruptive in the classroom is calling out of turn. The cause of this behaviour lies in the impulsive nature of ADHD and often functions as a way of seeking attention from the teacher or fellow pupils. In this sense, the response from the teacher or pupils reinforces further disruptive verbalisations, since the response provides the child with the attention it was seeking [11].

Since the above behaviours can disrupt the individual, teacher and other children in the class, there have been attempts to try to reduce these behaviours. Interventions include a range of cognitive behavioural techniques such as behaviour modification, cognitive training and social skills training. The most prevalent approach to reducing disruptive behaviours in the classroom is behavioural modification. Most behaviour modification techniques use operant conditioning [12], which focuses on manipulating the child's behaviour to improve it. Techniques found to be beneficial for children with ADHD involve a variety of reinforcers [13] such as: social (praising), tangible (tokens), activity (games) and punishers; removal of tokens (stickers) and privileges (break-time); or positive attention (time-out).

Despite the continued use of behaviour modification techniques to help reduce disruptive behaviour in the classroom, the approach itself has received much criticism. For example, the interventions require a great deal of time, social resources and patience to implement and require the consistent effort of teachers and parents [14]. Therefore, implementing successful behavioural interventions require clear guidelines and monitoring techniques, which has not always been the case for many studies. On a more fundamental level, such interventions only focus on observable, describable and measurable behaviour. However, the intricacies of behavioural problems in children with ADHD are not always made explicit. More crucially, behaviour modification techniques are often used in conjunction with medication; making it difficult to differentiate between whether success is as a result of the behavioural techniques or medication, or a combination of the two [15]. In an attempt to address these limitations, researchers continue to explore interventions that can be integrated into the school curriculum. One addition has been the Social Story [16].

School teachers, Gray and Garand [16] developed the Social Story (SS) to help Autistic children to better understand social situations and the thoughts and feelings of others within specific social situations. SS are individualised short stories designed to improve or teach social skills and positive social interactions. SS describe a situation, specific activity or behaviours related to social cues. The aim is to teach a specific skill to enable children to transfer the skills to other social settings by providing instructions on who, what, when, where and why of a social situation [17]. There are several benefits to using SS as an intervention within the classroom. For example, they present children with basic information about a particular situation that may be confusing to them. The SS also uses visual learning strategies which are beneficial to those with learning disabilities or prefer visual stimuli. SS are easy to implement as they do not require specialised training. Therefore, teachers can implement them into their daily routine without disruption to the schedule or curriculum. However, despite SS becoming an increasingly popular strategy across a wide range of behaviours, there are very few studies to-date that have used SS with ADHD children.

Despite the lack of research on ADHD using this intervention, there is extensive research that examines the effects of SS on the kinds of disruptive behaviour exhibited by children with ADHD. For example, in their study, Scattone, Wilczynski, Edwards, and Rabian [18] observed the disruptive behaviours of three children. One type of behaviour, calling out, disrupted the whole class since it led others to shout out. Following the SS intervention, all three children showed a reduction in their disruptive behaviours. The child who consistently called out showed a reduction in this behaviour from 16 per cent during the baseline trials to 5 per cent following the intervention. The rate of other children shouting out also decreased significantly. The authors praised the non-intrusive nature of SS and children's engagement with the stories (since reading is part of the child's daily task in school). However, the authors do acknowledge the influence of verbal prompts from teachers, and how these may have inflated the findings. This highlights the importance of providing teachers with clear guidelines on what they can and cannot do during the intervention period.

In 2011, Beh- Pajooh et al. [19] examined the effectiveness of SS in reducing the challenging behaviours of three boys with autism. They reported that for two out of three boys, challenging behaviour reduced in the classroom. In the same year, Benish and Bramlett [20] used SS on children with no developmental disorders, but who fell within the 'at-risk' from aggression on the Behaviour Assessment System for children-2 [21]. The authors reported that although SS were successful in decreasing aggression and increasing positive peer relations, they cite some methodological limitations. For example, there were environmental inconsistencies such as using different members of staff to work with individual children and a lack of monitoring the times of day each child received the SS. Furthermore, the researchers did not ask staff members whether they had used any additional interventions or praise at the time of the study. This lack of parity across observations may have affected the outcome due to differences in reinforcement and teacher characteristics such as presentation, styles and attitudes towards the study, the SS or the target child. This study highlights the need to acknowledge the importance of the role of the teacher in the implementation stage of the SS intervention. According to More et al. [22], the staff-pupil relationship factors highly on the success of the SS. Therefore, using a single member of staff that works closely with the child may increase the effectiveness of SS and extinguish some of the issues raised in Benish and Bramlett's [20] study.

In spite of the literature presented thus far showing success in reducing disruptive behaviour in the classroom, research on its overall efficacy is mixed. According to Kokina and Kern [23], SS are ineffective and their use questionable. Their study examined 18 studies, with 47 pupils. The authors reported SS either 
worked very well or not at all, $51 \%$ of the studies were highly effective, $44 \%$ ineffective and $4 \%$ questionable. Watt et al. [24] also reported poor results in his study to reduce challenging behaviours of six children with ASD. They found that the SS worked for only one child. Similarly, Reynhout and Carter [25] suggested that SS have a minimal clinical effect on behaviour. They state SS are attractive to practitioners due to their ease of implementation and inexpensiveness.

The majority of studies that demonstrate SS as an unsuccessful classroom intervention have included some methodological limitations. For example, some studies have used SS alongside other interventions, but have not factored this into their findings. These include operant conditioning (reinforcement), prompts and feedback during the intervention period $[18,26]$. Therefore, it is unclear whether it is the SS or other intervention that influences the behaviour changes observed [27]. Questions also arise surrounding the implementation of SS. In particular, the need to follow guidelines set out by Gray $[16,17]$. Following these guidelines at the creation stage and implementation is crucial for the integrity of SS and essential for training and planning purposes [28]. However, not all studies have followed the guidelines making any conclusions offered questionable. Kuoch and Mirenda [28] reported that in ten SS studies before 2003, 50 percent did not conform to Gray's guidelines, which highlight a potential issue of validity. Therefore, to ensure validity, the present study follows Gray's [17] SS guidelines.

Similarly, to address the lack of consistency highlighted in previous studies, the present study recruited one individual wellknown to each child to be involved at every stage of creation, monitoring and implementation. The best-placed person to do this was the classroom teaching assistant (TA). A TA provides support to an individual child or group, with the aim to support and augment behaviour strategies for children with emotional/ behavioural difficulties. Evidence suggests that TAs help improve confidence, motivation and on-task behaviour [29]. Since TAs spend a great deal of time one-to-one with pupils in less formal, personalised interactions [30], it would appear that TAs would be in the best position to deliver individual interventions. Research that supports the important role played by TAs in delivering successful interventions comes from Savage, Carless and Erten [31]. In their study on the effects of reading interventions, they found that experienced TAs helped two out of three children at risk of reading difficulties. Therefore, utilising TAs in the present study should provide a unique opportunity to monitor the individual's typical behaviour, administer the intervention and record any change to that behaviour following the intervention.

With the ever-increasing costs of ADHD to the individual, family and society $[32,33]$ there is a real need for alternative classroom interventions that are inexpensive, easy to implement and require little training. According to Telford et al. [34], ADHD leads to increased healthcare and education costs. In the UK alone, treatment costs are estimated at $£ 670$ million. Of this figure, $76 \%$ is spent on educational resources. Therefore, there are strong economic reasons to justify exploring more efficient strategies.

The present study aims to examine the efficacy of SS on children with $A D H D$, in light of the previous research and the methodological limitations highlighted here. It is hoped that the findings will help in the development of successful classroom interventions that can be implemented and monitored by TAs, which may go some way to improving the behaviours and outcomes for children with ADHD. It is predicted that children's disruptive behaviour (aggression, rising out of the seat and calling out of turn) will lessen following exposure to SS during a 10day intervention period. Furthermore, a decrease in disruptive behaviour in the days following the intervention (when the social story has been withdrawn) will highlight the potential for the long-term benefits of using SS in children with ADHD.

\section{Methodology}

\section{Design}

The study followed three boys with ADHD from three separate mainstream classrooms over a four-week period. For all three boys, the experiment comprised of four phases. In phase one, the pre-target behaviour phase, the TAs and classroom teachers completed the Conners' Rating Scales-Revised: Long Form (CTRS$\mathrm{R}: \mathrm{L},[35])$ and observed children's general disruptive behaviour in the classroom over a 5-day period. The target behaviour was established from the behaviour scale and initial observations. All target behaviours were recorded using the event sampling technique. The advantages of using event sampling over alternative data collection methods include the potential for rich, detailed descriptions that provide a practical way to observe the frequency and context of specific behaviours. Following this period the SS was created by the researcher. The second phase was to obtain a baseline by monitoring the frequency and extent of the target behaviour over a period of five days. The third phase was the intervention - the implementation of the SS for ten days. In the final phase, the SS was withdrawn, and the TA monitored any change in the target behaviour (compared with phase 2). Therefore, the dependent measures for each child consisted of the target behaviours that occurred prior, during and post-intervention. The independent variable was the SS. The TAs collected data for all four phases and was overseen by the classroom teacher.

\section{Participants}

Three boys from working-class backgrounds in south Wales, with an age range of 7.8 to 8.4 years, with ADHD, were selected for participation. The criteria for inclusion in the study included children from a mainstream school with a diagnosis of ADHD and a history of disruptive behaviour, and the ability to sit and read a book; and children allocated a TA who had experience using SS. All above criteria were fulfilled.

Johnny: Johnny (aged 8-years and four months) was diagnosed with ADHD at the age of 5. Johnny's intervention took place in his classroom of 22 children. The intervention was implemented by his TA, Sarah, who had worked with Johnny for the past two years. Sarah had used Social Stories on several occasions previously, but not with Johnny. For Johnny, aggression was identified as a problem behaviour that often disrupted his work 
and classmates. This behaviour occurred on numerous occasions during the observation period.

Observations revealed that Johnny was most likely to become aggressive after break and lunch times when he was expected to stop playing and receive instructions to start a new task. Therefore, Johnny's intervention took place after morning and afternoon break and after the lunch break. Johnny read his SS an average of 3 times-a-day for the first week, but this decreased to an average of twice a day after that. The decrease in engagement resulted from him becoming agitated and frustrated with the story and throwing it across the classroom.

Lee: Lee (aged seven year and eight months) was diagnosed with ADHD at the age of 6 . Lee's intervention took place in his classroom of 31 children. His TA, Maria implemented the intervention. Maria had worked with him for the past 18 months. Maria had used SS with previous children, but not with Lee. For Lee, getting out of his seat was identified as a problem behaviour that often resulted in disruption to the class and his work. Maria noted that the behaviour was worse when Lee was required to sit quietly and work by himself. This behaviour had implications for his academic work which often remained incomplete. Since Lee's target behaviour was not dependent on specific times during the day but occurred during the quiet-work time, he was presented with the SS before this period. Lee read his SS an average of 3 times-a-day.

Ben: Ben (aged eight years and five months) was diagnosed with $A D H D$ at the age of 4 . The setting for Ben's intervention was in his classroom of 20 children. Ben's TA, Sandy, began working with him in Nursery class and has remained with him to the present day. Sandy had used SS with previous children, but not with Ben. During phase one, it was noted that one particular behaviour stood out - calling out of turn. Ben would often shout out answers before being asked to answer the question posed to the class. He would also call out his teacher's name to get her attention, which would interrupt daily tasks and quiet working periods. Observations highlighted that Ben's calling out could occur at any time during the day and that it did not occur during specific time periods or tasks. Therefore, Ben read his SS at the start of each day and after lunch. Ben read his SS an average of 3 times-a-day.

In all three settings, non-target children continued with their daily activities and routines and were not part of the observations or study in any way.

\section{Materials}

The CTRS-R:L [35] consists of 59 questions designed for use in children aged 3-17 years. Most of the questions are based on behavioural characteristics that are described in the DSM-IV diagnostic guidelines for ADHD [36]. The scale is made up of seven sub-scales: oppositional; cognitive problems; hyperactivity; anxious-shy; perfectionism; and social problems. It also includes two global indices: restless-impulsive and emotional lability, and scales for overall functioning: ADHD index-DSM-IV: inattentive and hyperactive-impulsive symptoms. Each of the questions requires the teacher to score the child's behaviour from 0 to 3 where $0=$ not true at all/never, one=just a little true/occasionally, two=pretty much true/often, and 3=very much true/very often. The scale was completed initially before- and post-intervention. Initial scores were used to help identify the target behaviour. Post-intervention scores were compared with the initial scores to measure any change in behavioural characteristics.

Once the target behaviour had been established and documented, individual SS books were created by the researcher to reflect each child's target behaviour, interests, developmental level and abilities. The stories were created with each child's favourite video game or TV/Film characters in mind. Ben's story included pictures of Mario; for Lee, Harry Potter, and Johnny, Spiderman. Each story was designed following Gray's [17] four basic types of sentences; (a) descriptive, (b) coaching, (c) perspective, and (d) affirmative. Descriptive sentences provide information about the present environment. For example, statements of who is involved, where the situation takes place, what is happening, what is expected, and why. Coaching sentences gives the child specific instructions on how to respond to the situation, by using "I will try..." statements. Perspective sentences provide information about how others may be feeling or thinking. Affirmative sentences are used to help the child to remember the story better. Gray [17] stated that a SS should have a ratio of 2 to 5 descriptive, perspective, and/or affirmative sentences for every 0 to 1 coaching sentence. In the present study for every coaching sentence, there was between two to five other sentences in the story; this ensured that the story represented single target behaviours. A further necessity was to inform the TA not to provide feedback or prompts during the intervention period; this was to ensure that the only intervention being used was the SS. However, due to the important role reinforcement plays in teaching children with $A D H D$, the present study used internal (rather than external) reinforcers within each of the SS, i.e., 'my teacher will be happy if I...' (Perspective sentence). TAs received draft copies of the stories for feedback before final production. The SS books were created using Microsoft Publisher and printed on A5 paper, laminated and spiral bound into a booklet. There were eight pages in each SS.

\section{Procedure}

Two different data sets were gathered. First, a pre-post-test design was used to examine children's scores on the CTRS-R:L. The TAs completed the scale along with the classroom teacher before and after the study. The children's scores were then compared to see if any changes had occurred. The second data set was the frequency of target behaviours before, during and after the intervention. The children were observed every day for 20 days (four school weeks) during their regular, routine lessons.

Phase 1 - Observation period: Children were observed for five days using the event sampling technique, recording any behaviour that disrupted the class/teacher. This data, along with the information from the CTRS-R:L and discussions between the $T A$, classroom teacher and researcher was used to identify the target behaviour. Following this initial observation period, the researcher created the individual SS based on the information 
gathered

Phase 2 - Baseline target behaviour: Once the target behaviour was agreed by the TA and classroom teacher, a further five days of event sampling was carried out by the TA to obtain baseline measurements of the target behaviour before the intervention. The information gathered during this time determined the prevalence, when, where, with whom, at what times and during which activities the behaviour was most likely to arise. This information would inform the TA about the best time in which to implement the SS. Furthermore, information and frequencies of the target behaviour recorded at this stage were later compared to the data obtained post-intervention.

Phase 3-Intervention period: Children were presented with their individually tailored SS for ten days. The child's first encounter with the Story was presented by their TA and accompanied by five comprehension questions (i.e., what do all children in my class have to do before talking?). Each child was expected to answer the comprehension questions accurately. If this did not happen, the TA read the Story and answered the questions until the child could do so accurately. Ben and Johnny could answer the questions the first time they were introduced to their SS. Lee needed Maria to repeat the story and questions twice before answering them accurately. Once children had answered the comprehension questions correctly, they returned to their daily activity within the classroom. The TAs and teachers were advised not to give any form of prompt or reinforcement during the intervention period. The data was recorded and later compared to the data collected before the introduction of the SS.

Phase 4-Maintenance period: TAs removed the SS but continued with observations for a further five days after the intervention period had ended. The observations post-intervention would monitor whether or not children's target behaviour continued to decrease/change when the SS was no longer present.

\section{Data analysis}

Graphic representations and mean baseline reduction (MBR: [7]) were utilised to identify appropriate changes in target behaviour across the phases. The CTRS-R:L scores were initially converted into t-scores then analysed using a paired-sample t-test to observe any significant change in pre and post-intervention scores.

Inter-rater reliability was measured by assessing the levels of agreement and disagreement during approximately $30 \%$ of the observations for each participant during both baseline and intervention phases. The agreement included occasions when both observers, the TA and class teacher, agreed that the target behaviour occurred. Disagreements were scored if the observers did not agree that the target behaviour had occurred. Percentages of the inter-observer agreement for Johnny was 91\%, for Lee, $95 \%$ and Ben $99 \%$.

\section{Ethical Considerations}

The University of South Wales granted ethical approval. Consent was obtained from TAs, classroom teachers, Head of school and parents of the children. Each child was asked would they like to participate in the study. Parents were ensured that all personal information would be kept confidential and the only information taken from the observations would be written assessments. No photographs, audio or video recordings were made, and names were changed so that data remained anonymous.

\section{Results}

The following section presents the results for each of the three children. Target behaviours across the four weeks are depicted in Figures 1-3. These are followed by the individual results of the CTRS-R:L and qualitative data recorded by the TA and classroom teacher.

\section{Ben}

During the intervention periods, Ben read his SS at the start of each day and after lunch. Thus, reading the story 20 times in the 10-day intervention period. Figure 1 displays the results for Ben's target behaviour of calling out of turn and the introduction of raising his hand.

Figure 1 shows the average rate of calling out for Ben's baseline data was 6.8. This average decreased to 4.4 during the first week of intervention and continued to decrease in week two of the intervention (3.4). When the intervention was withdrawn in week 4 , the average rate of shouting out had decreased further to 0.8 . The range of Ben's calling out during the baseline and week one of the intervention was similar, ranging from 0 to 9 occasions at the baseline and 0 to 10 during week one of the intervention. Although the range decreased considerably by the second week of intervention (1 to 2 ) and post-intervention (0 to $3)$, variability here was similar. Thus, Ben's calling out decreased considerably from before intervention to post-intervention (6.8 to 0.8 , respectively).

To determine the effectiveness of the intervention the MBR was calculated by finding the mean baseline and mean intervention measurements for the last three data points in each. Then the mean of intervention was subtracted from the mean of baseline, divided by the mean of baseline and multiplied by 100. Percentages of 90 and above indicate a very effective intervention, 70-90\% indicate an effective intervention, 50-70, questionable and 50 and below indicate an ineffective treatment. Ben's MBR for calling out was $70 \%$, indicating the SS was an effective intervention for calling out of turn. Of interest is the new behaviour of raising his hand, as encouraged in his SS, in the second week of intervention. Figure 1 shows that Ben raised his hand before speaking an average of 3.4 times in week 2 , this behaviour increased considerably to 6.6 in week 4 , where the intervention had been withdrawn. This suggests that Ben had taken on board the information presented in the SS that promoted raising a hand to answer a question or to speak out.

Ben had talked about the information in the story during the day. He often referred to raising his hand before answering questions and that he should wait for his teacher to call on him for his answer. He also spoke with his TA when other children in his class answered questions before being asked for the answer - 


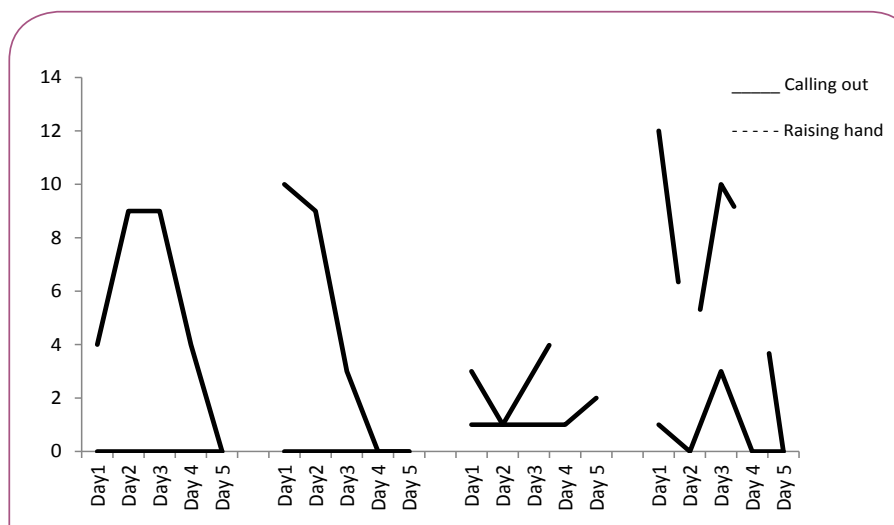

Figure 1 Frequency of calling out and raising hand over the 4 week observation period for Ben.
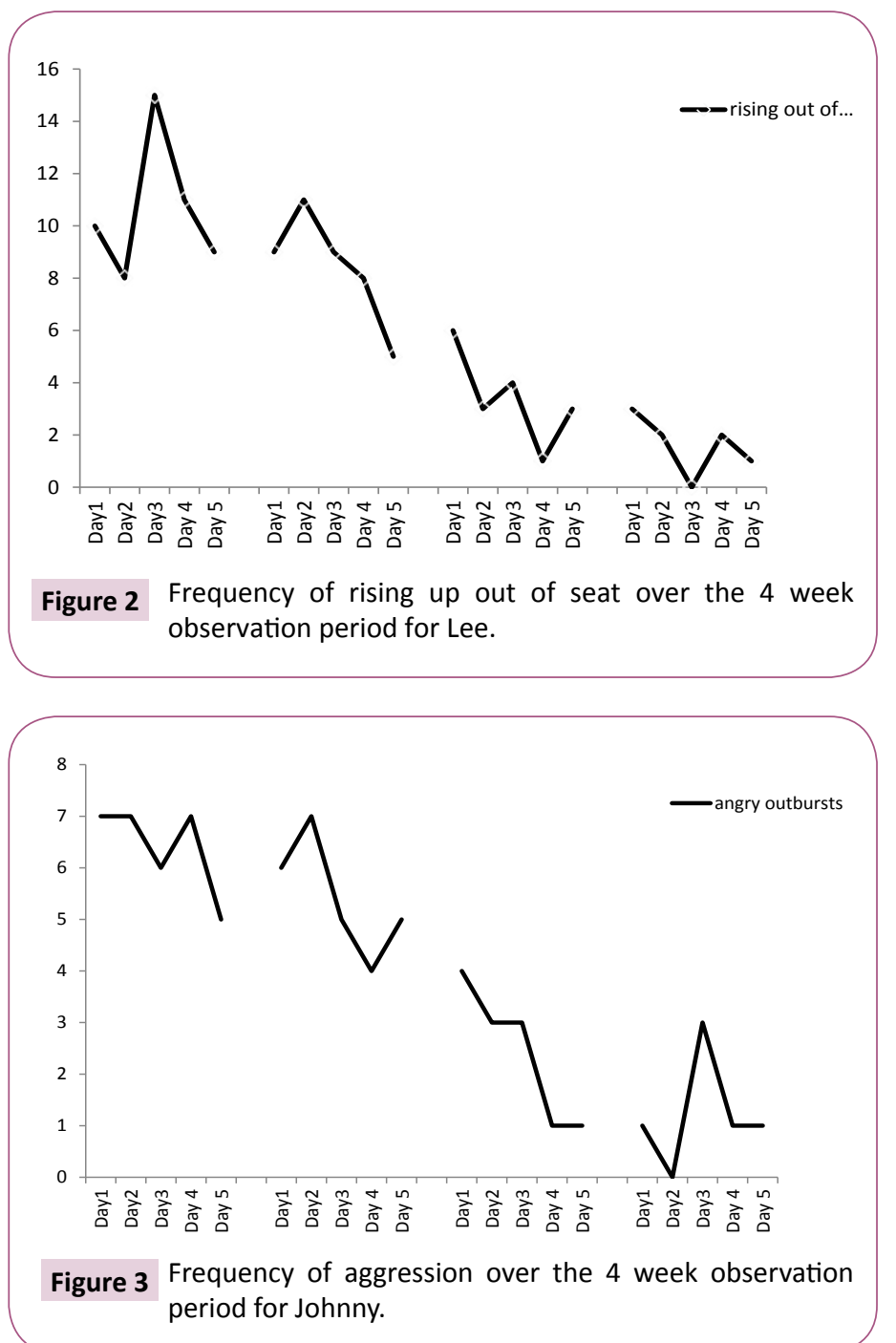

exclaiming 'they didn't put their hand up' and 'they are shouting out her [teacher] name, but don't have their hand up'.

Examination of the TA's notes surrounding the target behaviour revealed that all calling out occurrences took place during oral lessons/tests. This was not recognised as a pattern during the pre-baseline observations. However, the decrease in calling out suggests that Ben had learned how to behave appropriately during the oral teaching sessions. In addition to the quantitative results above, Ben's TA reported a vast improvement in his calling out. She explained that Ben was more aware of his actions when it came to answering questions. He would sometimes stop look at his TA and say 'I'm putting my hand up to answer this question' and 'I have to raise my hand if I want to speak to Miss'. Again, this supports the notion that Ben understood the consequences of his behaviour and that he had used an alternative strategy when wanting to speak out in class.

Anecdotal evidence from the classroom teacher revealed her surprise about the success of the intervention after such a short space of time. She had explained that the classroom was a great deal quieter now that Ben had reduced calling out of turn and had begun raising his hand. More importantly, she indicated that other children in the class prone to calling out had benefited, vicariously, from Ben's behaviour. The classroom teacher expressed her interest in creating further SS to use on the whole class.

\section{Lee}

During the intervention periods, Lee read his SS at the start of each day and before each quiet working period. Thus, Lee read his story 26 times in total. Figure $\mathbf{2}$ displays the results for Lee.

The average rate of rising out of the seat for Lee's baseline data was 10.6. This average decreased to 8.4 during the first week of intervention and continued to decrease in week two of the intervention (3.4). When the intervention was withdrawn at week 4 , the average rate had decreased further to 1.6. Similar to Ben's results, the continued decrease of rising out of seat over the four weeks suggests that the intervention affected the target behaviour. The range of Lee's rising out of the seat during the baseline and week one of the intervention was variable ranging from 8 to 15 occasions at the baseline and 5 to 11 during week one of the intervention. This variability decreased considerably by the second week of intervention ( 1 to 6 ) and post-intervention (0 to 3). Figure 2 shows that Lee's raising out of seat decreased considerably from before intervention to post-intervention (10.6 to 1.6 , respectively). Lee's MBR was 74 per cent, indicating the SS was an effective intervention for rising out of seat behaviour.

TA - event sampling: Data gathered from the event sampling observations revealed that at the end of the first week and during the second week of intervention, Lee repeated a number of the sentences from his SS throughout the day. In particular, he repeated 'when I' $m$ at the table I will sit on my chair with my feet on the floor' and 'sitting like this helps me learn'. Following these exclamations to his TA, Lee sought appraisal saying 'this is right, isn't it, sitting like this helps me learn and I get to finish my work.' This evidence supports the notion that Lee not only understood what the SS was asking of him but also the consequences of his actions. The TA noted that on three occasions during the intervention period, Lee stood up and motioned to walk around the classroom, but instead stopped looked at Maria or his work on the desk and sat back down quietly.

Anecdotal evidence from the classroom teacher revealed that she was not convinced that SS would be of benefit. However, 
following the intervention, she was delighted with the reduction in Lee's out of seat behaviour. She was particularly impressed with Lee's attitude towards remaining seated. She indicated that it was clear that he understood the importance of staying seated when completing his work, something that she had often tried to instil in him. Through his engagement with the SS, he had now learned that the reason he did not always complete his work, as his fellow pupils did, was a consequence of getting out of his seat continually.

\section{Johnny}

During the intervention period, Johnny read his SS after each break and lunchtime. However, in week two Johnny refused to read his SS three-times-a-day. Therefore, Johnny read his story 26 times in total. Figure 3 displays the results for Johnny.

The average rate of aggressiveness for Johnny's baseline data was 6.8. This average remained stable during the first week of intervention (6.8) and decreased slightly into week two of the intervention (5.4). When the intervention was withdrawn in week 4 , the average rate of aggressiveness had risen slightly to 5.6. In contrast to Ben's and Lee's results, Johnny's aggressiveness did not decrease substantiality over the four weeks suggesting that the intervention did not have a positive effect on his aggressiveness. The range of Johnny's aggressiveness during all four weeks remained low. For example, the baseline and week one of the intervention ranged from 5 to 9 occasions. A similar range continued through the second week of intervention (3 to 7) and post-intervention (4 to 7). Figure 3 shows that Johnny's aggressiveness decreased slightly from before intervention to post-intervention (6.8 to 5.6, respectively), but not enough to make a significant change. Johnny's MBR was 30 per cent, indicating the SS was an ineffective intervention for aggressiveness.

TA - event sampling: Johnny's TA reported that although initially, Johnny engaged with his SS in week one, engagement rapidly decreased in the following two weeks. Johnny became increasingly frustrated with the SS and refused to read it on some occasions (usually after lunch). It was also noted that on numerous occasions Johnny threw the SS across the classroom calling it a stupid story. However, Johnny's TA reported that for most aggressiveness that continued into the second and third week of the study, Johnny repeated some sentences from his SS. For example, he said 'It is okay to be angry everybody gets angry because I'm not hurting anyone, right'? This indicated that he understood the relationship between his behaviour and the information presented in the SS. Furthermore, despite no decrease in his aggressiveness, his hitting out at his fellow pupils and TA decreased considerably, showing that the SS was effective for this associated behaviour.

Anecdotal evidence from the classroom teacher reported that although the SS was not successful in decreasing the target behaviour of aggressiveness, she had seen a distinct change in his attitude. She commented on the fact that Johnny now understood that he was aggressive, something that he hadn't acknowledged before. Johnny's classroom teacher indicated that this was a huge step forward for Johnny. She expressed her interest in continuing using the SS with Johnny, believing that with continued exposure Johnny could reduce his aggressiveness.

The CTRS-R:L was used to obtain a general classroom behavioural profile for each of the three children. Teachers completed the scale before and after the study. A paired-sample t-test was used to observe any significant change across pre and postintervention. Although there were some small changes noted across a number of sub-scales (in particular, for Ben and Lee on the hyperactivity, restless-impulsive global index and DSMIV: Hyperactive-Impulsive subscales), the results of the t-tests revealed no significant changes in the general classroom behaviour in pre- and post-test scores ( $p>0.05$, for all three comparisons).

\section{Discussion}

The present study makes a unique contribution to the SS literature in its use of children with ADHD. The study aimed to examine the efficacy of SS on three boys with ADHD. It was predicted that target behaviours would decrease following SS intervention and continue a week after the SS had been withdrawn.

The results indicate that SS intervention was effective in reducing the disruptive target behaviour with two out of the three children with ADHD. Following SS implementation, Ben and Lee showed a reduction and consistent rates (less variability) in their target behaviours compared to their baseline rates. This reduction in target behaviour and variability continued into week four, as predicted. Further success was found in Ben's new behaviour of raising his hand before answering questions or speaking out. Evidence from Ben's TA supports an increase in this behaviour as he often expressed concern that other children were calling out without raising their hands. Similarly, anecdotal evidence from Lee's classroom teacher provided insights into the success of his reduced target behaviour and welcomed the significant shift in his attitude to remaining seated and his acknowledgement that getting out of his seat impacted continuously on his ability to complete his work. These findings have significant implications for children with ADHD. A key characteristic of the disorder is an inability to understand consequences due to a lack of hindsight and foresight [1]. Thus, the fact that Ben and Lee could understand the consequences following the intervention suggests that SS may help to negate the deficits associated with hindsight and foresight.

Although the SS was successful in reducing the target behaviours for Ben and Lee, the same effects were not found for Johnny. There are several reasons why this may have occurred. First, the coaching sentences, for example, count to ten and jump up and down were strategies that had been used with Johnny before the SS, but had failed. Therefore, it may be the strategy was too weak to prompt change. Another reason may be linked to reinforcement. Johnny continually sought appraisal and reinforcement during the intervention period. Therefore, the inherent nature of the reinforcements presented within the SS was not sufficient to help Johnny reduce his aggressiveness. Johnny's classroom teacher also indicated that he might need 
more time with his SS to absorb what is expected of him before he can respond appropriately. Therefore, if Johnny had received reinforcement and further exposure to his SS, the outcome may have been different.

The results support findings from some previous studies that have demonstrated a reduction in disruptive behaviour following SS $[18,19]$. The findings also support previous studies [37] that indicate the effectiveness at the maintenance stage (at week 4). Maintenance data is crucial when examining any long-term benefits of SS.

The CTRS-R:L was used to determine the behavioural profiles for all three boys pre- and post-intervention. Results revealed no significant change. One reason could be that the SS only targeted one area of difficulty on the CTRS-R:L. Another reason may be related to the short time frame used, just four weeks between each measurement. Therefore, future research may need to monitor some difficulties within the CTRS-R:L, and increase the time between presentations of the CTRS-R:L to observe any significant change.

The present study made a significant contribution relating to the omission of prompts and reinforcement during the observation period. Previous studies $[20,26]$ have failed to account for the presence of contributing interventions which affect the internal validity of the study. The finding that target behaviours decreased in two of the children suggests that SS alone are sufficient to elicit behavioural change. A further strength of the present study lies in the use of real-life target problems. These were issues that each child faced on a daily basis and where previous strategies had failed, making the study not only ecologically valid but also ameliorates previous concerns of a mismatch between the intervention and the skill deficit of the child [38]. An essential consideration in the present study was adherence to Gray's [17] SS guidelines. Many previous studies that have reported poor outcomes have not always followed them [28]. The effectiveness of the SS in the present study may be attributable to this. Following the guidelines also adds to the validity of the study and provides schools with a framework from which to work.

The rationale for using TAs in the present study was two-fold. First, it was hoped that the TA would help to reduce the disruption caused to the classroom, and second, to ensure that each child felt at ease with the person implementing the intervention and observing them. To date, there has only been one other study [39] that has used TAs in all elements (choice of target behaviour, implementation and observations). The success of the present study may be attributable to this in some way since the children were reported to behave normally throughout the study. Furthermore, given that the TA used event sampling to obtain data, the researcher could obtain a full picture of when exactly the behaviour occurred during the day. This allowed the researcher to target these behaviours at the right times, which not only added to the validity of the SS but also provided some information on the function of each of the target behaviours.

The study offers several implications for practice. Along with most studies that evaluate the use of SS, the present study investigated their efficacy in decreasing challenging behaviours only. However, in one classroom the SS was effective in increasing positive behaviours in the individual and on the class as a whole. Therefore, educators may wish to incorporate SS as a whole classroom approach to increasing pro-social behaviours. Furthermore, since reading is a natural part of a child's school day and can be implemented across several situations, at any time during the school day, the SS offers an unobtrusive intervention that can be used to increase opportunities to teach and reinforce specific social skills. The change in behaviour in the present study occurred relatively quickly. Schools are continually looking for quick and effective strategies that can be implemented without too much disruption to the everyday running of the classroom. Therefore, the 4-week period that elicited change in the present study may offer teachers a rapid and effective, alternative classroom intervention. A final implication for classroom practice was the success of using SS without prompts or reinforcement. Most classrooms rely heavily on reinforcement techniques, and the finding that SS were successful without them may encourage educators to look beyond behaviour modification when trying to reduce disruptive behaviour in their classrooms.

Consideration must be given to several limitations in this study. The primary limitation is that the SS showed a behavioural change in only two of the three children. Although the change in Ben and Lee's behaviour tells us that SS are successful for some children with ADHD, the lack of change in Johnny's behaviour limits the conclusions that can be drawn about the efficacy of SS for ADHD. The small sample of boys in a single age group makes generalising the findings difficult. Future researchers may wish to increase their participant size and include girls and different age cohorts. Although the present study gathered background information on each child before participation (history, CTRS:R-L and strict inclusive criteria), there were some critical omissions which may affect replicability. These include language and cognitive measurements and the severity and type of ADHD exhibited. Future studies may wish to explore these factors further to determine whether SS are suitable for different ability/severity levels. Furthermore, no comparison data was collected that compared the behaviours of those who took part with that of their peers. This does not allow the researcher to determine the extent to which external forces within the classroom contribute to the disruptive behaviours (outside of ADHD symptoms). Thus, future researchers may benefit from gathering social comparison data as well as individual baseline comparisons.

An essential element of the present study was to monitor the effectiveness of SS without additional interventions. However, although prompts and reinforcement were not part of the methodology, teachers have a natural tendency to use these strategies to help children learn and reach their goals. Moreover, since the researcher was not present at all observations, it was not possible to verify the use of prompts or reinforcement. Therefore, future research should strive to control the use of additional strategies by implementing an $A B A C B C$ design that explores the influence of each component (i.e., prompts versus no prompts). 


\section{Conclusion}

The purpose of this study was to assess the efficacy of SS in children with ADHD. Results indicate effectiveness in reducing the disruptive behaviours in two out of the three children. Furthermore, reduction continued once the SS had been withdrawn suggesting that children had learned appropriate social responses following the intervention. Although SS show

\section{References}

1 Barkley RA (2014) Attention-deficit hyperactivity disorder: A handbook for diagnosis and treatment. Guilford Publications.

2 NICE (2013) CG72 Attention deficit hyperactivity disorder (ADHD) guideline.

3 Barkley RA (2006) The relevance of the still lectures to attentiondeficit/hyperactivity disorder: a commentary. J Atten Disord 10: 137 140.

4 Loe IM, Feldman HM (2007) Academic and educational outcomes of children with ADHD. J Pediatr Psychol 32: 643-654.

5 Barbaresi WJ, Katusic SK, Colligan RC, Weaver AL, Jacobsen SJ (2007) Long-term school outcomes for children with attention-deficit/ hyperactivity disorder: a population-based perspective. J Dev Behav Pediatr 28: 265-273.

6 Martin AJ (2012) The role of personal best (PB) goals in the achievement and behavioural engagement of students with ADHD and students without ADHD. Contemp Educ Psychol 37: 91-105.

7 Campbell SB, Von Stauffenberg C (2009) Delay and inhibition as early predictors of ADHD symptoms in third grade. J Abnorm Child Psychol 37: 1-15.

8 Montague M, Castro M (2005) Attention deficit hyperactivity disorder: concerns and issues. Handbook of Emotional \& Behavioural Difficulties 399-415.

9 Ohan JL, Visser TA, Strain MC, Allen L (2011) Teachers' and education students' perceptions of and reactions to children with and without the diagnostic label "ADHD". J Sch Psychol 49: 81-105.

10 Bennett D, Pitale M, Vora V, Rheingold AA (2014) Reactive vs. proactive antisocial behavior: differential correlates of child ADHD symptoms. J Atten Disord 7: 197-204.

11 Chandler LK, Dahlquist CM (2014) Functional assessment: Strategies to prevent and remediate challenging behavior in school settings. Pearson Higher Education, p: 352.

12 Skinner BF (1992) "Superstition" in the pigeon. J Exp Psychol Gen 121: 273.

13 Antshel KM, Hargrave TM, Simonescu M, Kaul P, Hendricks $K$, et al. (2011) Advances in understanding and treating ADHD. BMC Med 9: 72.

14 Martens BK, Witt JC, Elliott SN, Darveaux DX (1985) Teacher judgments concerning the acceptability of school-based interventions. Professional Psychology: Research and Practice 16: 191.

15 Musser EH, Bray MA, Kehle TJ, Jenson WR (2001) Employing precision requests and antecedent strategies to reduce disruptive behaviour in students with social and emotional disorders: A replication. Sch Psychol Review 30: 294-304.

16 Gray CA, Garand JD (1993) Social stories: Improving responses of potential to be an effective approach for children with ADHD, further replication with consideration given to the limitations outlined is needed.

\section{Conflict of Interest}

No conflict declared.

students with autism with accurate social information. Focus on Autistic Behaviour 8: 1-10.

17 Gray C (2010) The new social story book. Future Horizons.

18 Scattone D, Wilczynski SM, Edwards RP, Rabian B (2002) Decreasing disruptive behaviours of children with autism using social stories. $J$ Autism Dev Disord 32: 535-543.

19 Beh-Pajooh A, Ahmadi A, Shokoohi-Yekta M, Asgary A (2011) The effect of social stories on reduction of challenging behaviours in autistic children. Procedia Soc Behav Sci 15: 351-355.

20 Benish TM, Bramlett RK (2011) Using social stories to decrease aggression and increase positive peer interactions in normally developing pre-school children. Educ Psychol Pract 27: 1-17.

21 Reynolds C, Kamphaus R (2004) BASC-2 Behavior assessment for children manual. Circle Pines, MN: American Guidance Service.

22 More CM, Sileo NM, Higgins K, Tandy RD, Tannock M (2013) The effects of social story interventions on preschool age children with and without disabilities. Early Child Dev Care 183: 1-16.

23 Kokina A, Kern L (2010) Social story ${ }^{\mathrm{TM}}$ interventions for students with autism spectrum disorders: A meta-analysis. J Autism Dev Disord 40: 812-826.

24 Watt N, Wetherby AM, Barber A, Morgan L (2008) Repetitive and stereotyped behaviors in children with autism spectrum disorders in the second year of life. J Autism Dev Disord 38: 1518-1533.

25 Reynhout G, Carter M (2011) Evaluation of the efficacy of social stories $^{\mathrm{TM}}$ using three single subject metrics. Res Autism Spectr Disord 5: 885-900.

26 Kalyva E, Agaliotis I (2009) Can social stories enhance the interpersonal conflict resolution skills of children with LD? Res Dev Disabil 30: 192-202.

27 Ali S, Frederickson N (2006) Investigating the evidence base of social stories. Educ Psychol Pract 22: 355-377.

28 Kuoch H, Mirenda P (2003) Social story interventions for young children with autism spectrum disorders. Focus Autism Other Dev Disabl 18: 219-227.

29 Webster RA, Russell P, Blatchford P (2012) Reassessing the impact of teaching assistants: How research challenges practice and policy. Routledge.

30 Rubie-Davies C, Blatchford P, Webster R, Koutsoubou M, Bassett P (2010) 'Enhancing student learning? A comparison of teacher and teaching assistant interaction with pupils'. Sch Eff Sch Improv 21: 429-449.

31 Savage R, Carless S, Erten O (2009) The longer-term effects of reading interventions delivered by experienced teaching assistants. Support for Learning 24: 95-100. 
32 Hakkaart-van Roijen L, Zwirs BWC, Bouwmans C, Tan SS, Schulpen TWJ, et al. (2007) Societal costs and quality of life of children suffering from attention deficit hyperactivity disorder (ADHD). Eur Child Adolesc Psychiatry 16: 316-326.

33 de Graaf R, Kessler RC, Fayyad J, ten Have M, Alonso J, et al. (2008) The prevalence and effects of adult attention-deficit/hyperactivity disorder (ADHD) on the performance of workers: results from the WHO World Mental Health Survey Initiative. J Occup Environ Med 65: 835-842.

34 Telford C, Green C, Logan S, Langley K, Thapar A, et al. (2013) Estimating the costs of ongoing care for adolescents with attentiondeficit hyperactivity disorder. Soc Psychiatry Psychiatr Epidemiol 48 : 337-344.
35 Conners CK (1997) Conners' Teacher Rating Scale-Revised (L). MultiHealth Systems North Tonawanda, NY.

36 APA (2001) The principles of medical ethics: With annotations especially applicable to psychiatry: Amer Psychiatric Pub Incorporated.

37 Crozier S, Tincani M (2007) Effects of social stories on prosocial behavior of preschool children with autism spectrum disorders. J Autism Dev Disord 37: 1803-1814.

38 Quinn MM, Kavale KA, Mathur SR, Rutherford Jr RB, Forness SR (1999) A meta-analysis of social skill interventions for students with emotional or behavioral disorders. J Emot Behav Disord 7: 54-64.

39 Toplis R, Hadwin JA (2006) Using social stories to change problematic lunchtime behaviour in school. Educ Psychol Pract 22: 53-67. 